\title{
BMJ Open Is it necessary for children to receive professional fluoride in addition to regular fluoride toothpaste? Protocol for a systematic review
}

\author{
Lintong Yu, ${ }^{1}$ Xueqian $\mathrm{Yu}^{2}{ }^{2}$ Yueyang Li, ${ }^{3}$ Jun $\mathrm{Li}^{2}$ Fang Hua (D) , ${ }^{4,5}$ Guangtai Song (1) ${ }^{1}$
}

To cite: Yu L, Yu X, Li Y, et al. Is it necessary for children to receive professional fluoride in addition to regular fluoride toothpaste? Protocol for a systematic review. BMJ Open 2020;10:e037422. doi:10.1136/ bmjopen-2020-037422

- Prepublication history for this paper is available online. To view these files, please visit the journal online (http://dx.doi. org/10.1136/bmjopen-2020037422).

$\mathrm{LY}$ and $\mathrm{XY}$ contributed equally.

Received 07 February 2020

Revised 29 June 2020

Accepted 10 August 2020
Check for updates

(C) Author(s) (or their employer(s)) 2020. Re-use permitted under CC BY-NC. No commercial re-use. See rights and permissions. Published by BMJ.

For numbered affiliations see end of article.

\section{Correspondence to}

Dr Fang Hua;

huafang@whu.edu.cn and

Professor Guangtai Song;

gtsong@whu.edu.cn

\section{ABSTRACT}

Introduction Regular toothbrushing with fluoride toothpaste is a fundamental intervention for caries prevention. Professional fluoride application (PFA) is widely considered a beneficial supplement to the routine use of fluoride toothpaste. However, some recent studies have failed to demonstrate the preventive effect of PFA. In addition, an increasing number of studies have highlighted the potential adverse effects of fluoride. However, little information exists on the effectiveness of additional PFA. The objective of this review is to systematically analyse the efficacy of PFA in addition to regular fluoride toothpaste among children under the age of 16 .

Method and analysis We will search the PubMed, Embase, Google Scholar and Cochrane Central Register of Controlled Trials databases for randomised controlled trials without language or publication date restrictions. Additional studies will be identified by manually searching the reference lists of the included studies and relevant reviews. At least two authors will carry out the selection of studies independently and in duplicate. The Cochrane Risk of Bias tool will be used to assess the risk of bias of the included studies. The random effects model will be used for meta-analyses. The data synthesis will be conducted using Review Manager software (RevMan V.5.3). The Grading of Recommendation, Assessment, Development and Evaluation will be used to assess the quality of supporting evidence for each major comparison.

Ethics and dissemination There is no need for ethical approval. The results of this review will be disseminated through peer-reviewed publications and social networks. PROSPERO registration number CRD42020165270

\section{INTRODUCTION}

\section{Background}

Dental caries is one of the most prevalent chronic diseases of people worldwide. ${ }^{1}$ If dental caries are not treated in time, the related consequences of tooth pain, inflammation and even premature tooth loss will affect children's growth, learning and quality of life and will lead to an increased economic burden for society. ${ }^{2}$

Fluoride toothpaste has been widely used for more than three decades and remains the
Strengths and limitations of this study

- Only randomised controlled trials will be included, as these studies are more likely to provide unbiased information than other study designs.

- Subgroup and sensitivity analyses will be performed to explore heterogeneity and the robustness of our findings.

- The reliability of the results will largely depend on the quantity and methodological quality of the primary studies included in this review.

standard intervention for preventing caries. ${ }^{2}$ For self-care, fluoride toothpaste is the most powerful intervention because of its clinical effectiveness and social acceptability. ${ }^{3}$ Most children use either sodium monofluorophosphate or stannous fluoride, usually at a concentration of 1000 parts per million (ppm) F, followed by sodium fluoride and other fluoride-containing formulations. ${ }^{2}$ The caries-preventive effect is statistically significant only for concentrations of 1000 ppm and above. ${ }^{4}$ Many professional associations have already endorsed the recommendation as a consensus: based on the best available evidence, all children should use standard fluoride toothpaste $(\geq 1000 \mathrm{ppm})$, regardless of their age ${ }^{45}$ The best way to reduce the side effects of fluoride is to reduce the amount of fluoride toothpaste that is ingested rather than adjusting the fluoride concentration. ${ }^{5}$

On the other hand, the Guideline on Fluoride Therapy recommends the professional application of topical fluoride treatment as an effective way to reduce caries in children who are at risk for this disease. ${ }^{6}$ However, the addition of professional fluoride application (PFA) to the daily use of fluoride toothpaste may lead to inefficacy due to repeated use. ${ }^{7}$ Especially in preschoolers, the risk of ingestion and subsequent dental fluorosis should be considered. ${ }^{89}$ When fluoride is used with 
other fluorine-containing vehicles, care must be taken regarding the cumulative fluoride exposure of children under 6 years of age. ${ }^{4}$ Additionally, cost-effectiveness must be considered for groups with low caries prevalence. ${ }^{10}$

Regarding efficacy and adverse effects, is it necessary for children to receive PFA in addition to regular fluoride toothpaste? There have been some related original studies in recent years, but no systematic review has yet been performed.

\section{Description of the intervention}

Dentistry has been successful in preventing dental caries through community, professional and individual preventive measures. ${ }^{11}$ The use of fluoride toothpaste and mouth rinse can be carried out by children's parents or by themselves as an individual measure. ${ }^{12}$ Toothbrushing is usually carried out using a manual or powered toothbrush and a fluoride toothpaste for 2 min two times a day. ${ }^{2}$ Systematic reviews have shown that only toothpastes with fluoride concentrations of at least $1000 \mathrm{ppm}$ can prevent caries effectively. ${ }^{4}$ The typical fluoride concentration of regular toothpaste is approximately 1000-1500 ppm. ${ }^{2}$ Unlike individual measures, PFA is usually carried out by professional dentists or participants, including forms of gel, foam and varnish. ${ }^{13} 14$ The typical concentration used in PFA is $12300 \mathrm{ppm}$ or $22600 \mathrm{ppm}$, which is much higher than the concentration used in individual use. ${ }^{11}$ The intervention of PFA at different concentrations at different frequencies can be combined with the use of fluoride toothpaste.

\section{Why is it important to conduct this review?}

First, the widespread availability of fluoride from multiple sources has raised the question of whether additional PFA is safe and effective to reduce caries. ${ }^{15}$

Second, the extent to which PFA in addition to the regular use of fluoride toothpaste provides extra protection against caries is unclear; this issue is of clear importance and needs to be formally investigated. ${ }^{16}$

Additionally, the Cochrane library contains only six systematic reviews and meta-analyses regarding fluoride use $^{16-21}$; however, only one review, published in 2004, mentioned a combination of toothpaste and PFA. ${ }^{16}$ That article did not take the concentration of fluoride in toothpaste into consideration, and its evidence remains to be updated. To the best of our knowledge, there has been no systematic review regarding this specific question.

\section{Objectives}

The main objective of our review is to assess whether there is a beneficial effect of adding PFA to regular fluoride ( $\geq 1000 \mathrm{ppm}$ ) toothpaste for children under 16. Additionally, we will assess whether PFA has some adverse effects.

\section{Review question}

Is it more effective for children under 16 to receive PFA in addition to regular fluoride toothpaste compared with using regular fluoride toothpaste alone? Additionally, are there any adverse effects of PFA?

\section{METHODS}

The protocol was written in accordance with the Preferred Reporting Project for Systematic Reviews and Meta Analyses (PRISMA) Protocol reporting guideline. ${ }^{22}$

\section{Criteria for considering studies for this review \\ Types of studies}

We will include randomised controlled trials (RCTs) in which:

1. PFA (with fluoride in any form or concentration) was used in addition to fluoride toothpaste and the concentration of fluoride in toothpaste was reported ( $\geq 1000 \mathrm{ppm}$ ).

2. The follow-up period (from the first intervention) was at least 6 months.

Types of participants

We will include studies in which the majority (over $80 \%$ ) of children are under the age of 16 at the time of recruitment. For consistency with existing systematic reviews on topical fluorides, participants aged 16 or under at the start of a trial will be classified as children. ${ }^{23} 24$

\section{Types of interventions}

The intervention of interest will be as follows:

- Combined use of PFAs (with fluoride in any form or concentration) and regular toothbrushing with regular fluoride toothpaste $(\geq 1000 \mathrm{ppm})$.

The comparison of interest will be/include:

- Self-applied toothpaste with a concentration of at least 1000 ppm alone.

Types of outcome measures

\section{Primary outcomes}

1. Changes in decayed, missing and filled surfaces or teeth (DMFS/DMFT in permanent teeth and dmfs/ $\mathrm{dmft}$ in deciduous teeth, continuous outcome, measured at least 6 months after application).

2. Any adverse effects related to fluoride, such as dental fluorosis, allergic reactions and fluorosis.

\section{Secondary outcomes}

1. Progression of caries lesion through enamel or into dentine and caries arrest international caries detection and assessment system (ICDAS/ICDAS II) or DIAGNOdent, continuous outcome, measured at least 6 months after application).

2. Patient-reported outcomes (eg, ease of use/quality of life).

3. Time taken for application.

4. Cost of treatments.

\section{Search strategy}

Electronic searches

We will identify relevant RCTs by searching the PubMed, Embase, Google Scholar and Cochrane Central Register of Controlled Trials databases without language or publication date restrictions. Table 1 lists the detailed search strategy for MEDLINE, which is tailored to the syntax 


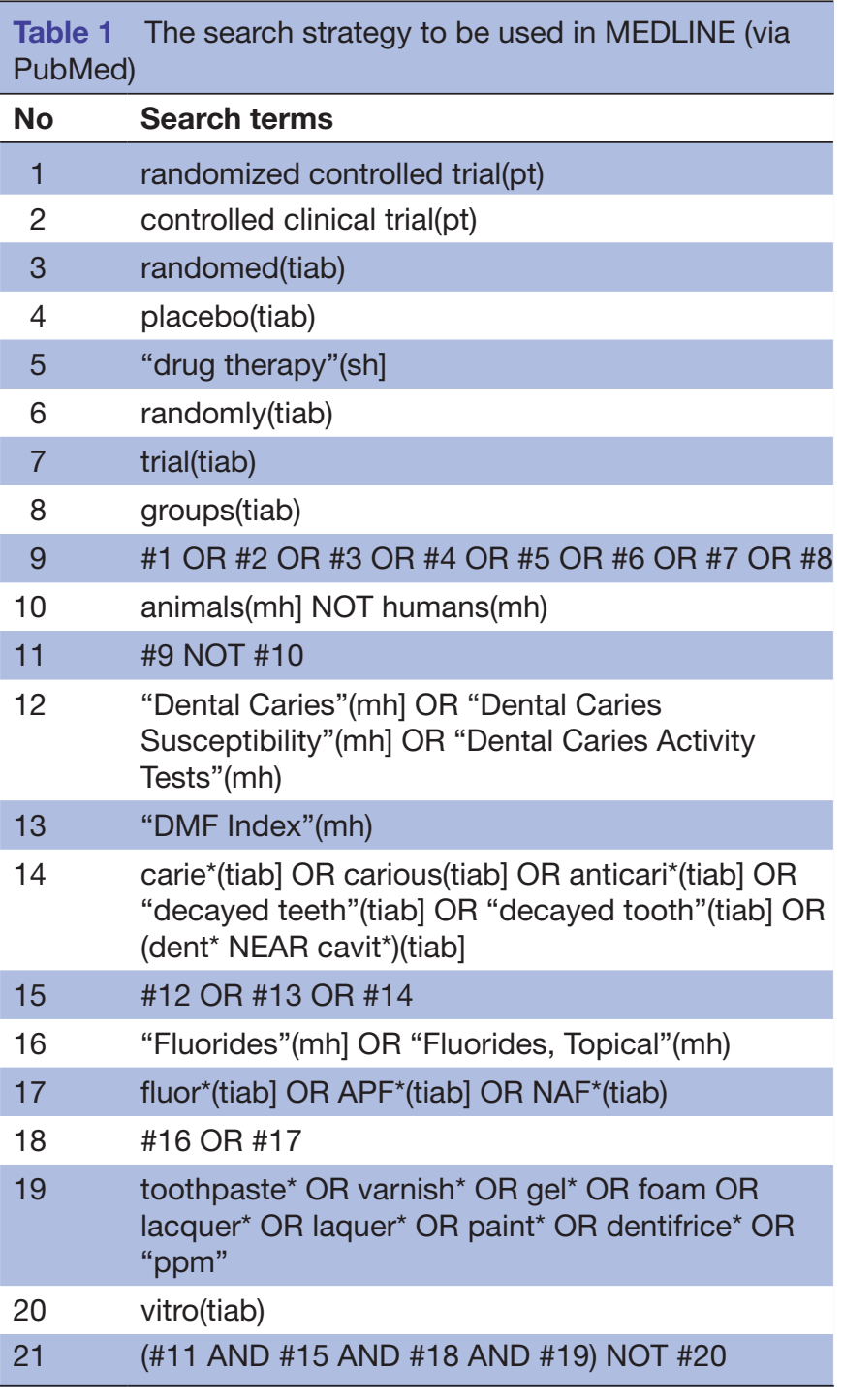

and topics of other databases. The subject search will be linked with the highly sensitive search strategy designed by Cochrane for identifying RCTs. ${ }^{22}$

\section{Identification of other studies}

Additional studies will be identified by manually checking the reference lists of the included studies and relevant reviews.

\section{Data extraction and analysis}

We will follow the guidance provided in the Cochrane Handbook for Systematic Reviews. ${ }^{22}$

\section{Selection of studies}

At least two authors will independently carry out the selection of studies and make decisions about eligibility. If the relevance of a study report is unclear, we will review the full text and resolve all disagreements by discussion. If consensus cannot be reached through discussion, a third reviewer serving as the arbitrator will decide whether a particular publication meets our eligibility criteria.
Data extraction and management

At least two authors will independently extract data and resolve discrepancies by consensus. The extracted data will consist of six components:

1. General information: title, publication year, countries where the studies were carried out, journal and author information.

2. Study characteristics: sample size, date and duration of study, method for random selection, allocation concealment, blinding.

3. The patient characteristics: age range, gender, dentition (primary, mixed or deciduous dentition), caries risk, clinical features (eg, location of the lesions) and demographic features of the individuals at baseline.

4. Intervention: type of intervention and type of control, application interval, fluoride content of toothpaste, other sources of fluoride, and other measures to prevent caries.

5. Outcomes: the instrument or scale for measurement, detailed description of the outcomes of interest (both beneficial and adverse).

6. Results: number of patients, point estimates and measures of variability for continuous variables, frequency counts for dichotomous variables.

\section{Risk of bias assessment}

Two authors will assess the risk of bias about the included studies dependently and in duplicate using the Cochrane Risk of Bias tool. ${ }^{22}$ The tool addresses the seven following key domains: sequence generation, allocation concealment, blinding of participants and personnel, blinding of assessment, incomplete outcome data, selective reporting and other bias. RCTs will be judged as having low, high or unclear risk of bias. We will compare and discuss the independent assessments of risk of bias with a third author to resolve any disagreement.

\section{Measures of treatment effect}

When we identify an adequate sample of studies with homogeneous populations and characteristics, we will get a meta-analysis of primary and secondary results. We will express dichotomous outcomes as risk ratios (RR) with $95 \%$ CIs and express continuous outcomes as mean differences (MD) with 95\% CIs. If the included studies assess common continuous outcomes using different scales, we will present the standardised MD (SMD) as a summary statistic.

\section{Missing data}

We will contact the corresponding authors to obtain any missing information or data if necessary. If we find instances of missing SDs, we will impute these data according to guidance given in the Cochrane Handbook for Systematic Reviews of Interventions. ${ }^{22}$ In addition, we will discuss the potential impact of missing data in the review. 


\section{Data synthesis}

As recommended by the Cochrane Collaboration, ${ }^{22}$ data synthesis will be conducted using Review Manager software (RevMan V.5.3). Meta-analyses will be performed where homogeneity across participants, interventions and outcomes is found. We will combine MDs or SMDs for continuous data and RRs for dichotomous data. Generally, we will apply a random-effects model to analyse pooled data, as the CI of the mean effect size will be wider than that obtained from a fixed effects model and will consequently lead to a more conservative interpretation. ${ }^{25}$

\section{Subgroup analyses and investigation of heterogeneity}

If possible, subgroup analyses will be conducted to explore possible sources of heterogeneity. ${ }^{26}$ We will try to perform subgroup analyses based on the following group distinctions:

1. The interval of PFA.

2. Different dentitions of primary, mixed or deciduous dentition.

3. Length of follow-up.

4. Levels of caries risk. ${ }^{27}$

\section{Sensitivity analyses}

If there are a sufficient number of studies included in this review, we will perform sensitivity analyses to assess the robustness of our results by excluding studies with a high risk of bias in the sequence generation and allocation concealment domains.

We will perform sensitivity analyses to compare effect estimates obtained by the fixed effects and randomeffects models. If these estimates lead to different interpretations of the data, we will report both models and attempt to investigate the difference.

\section{Assessment of publication bias}

Possible publication bias will be estimated by assessing the small study bias through a funnel plot and Egger's test if at least 10 studies are included in the meta-analysis. ${ }^{28}$

\section{Summary of findings tables}

The Grading of Recommendation, Assessment, Development, and Evaluation (GRADE) framework will be used to assess the quality of supporting evidence behind each major comparison. ${ }^{29} 30$ RCTs will start with a high certainty of evidence. Thereafter, five factors (risk of bias, imprecision, inconsistency, indirectness and publication bias) may lead to downgrading the certainty of evidence, and three factors (large effect, dose response and all plausible confounding would reduce a demonstrated effect) may lead to upgrading the certainty of evidence.

\section{Patient and public involvement}

The current work is based on a review of the studies and does not include original patient data. Therefore, there is no patient or public involved in our review protocol.

\section{ETHICS AND DISSEMINATION}

There is no need for ethical approval, and the results of the review protocol will be disseminated through peerreviewed publications and social networks.

\section{DISCUSSION}

This review will assess the possible evidence about whether there is a beneficial effect of adding PFA to the use of regular fluoride $(\geq 1000 \mathrm{ppm})$ toothpaste. We will perform the literature search, data extraction, and risk of bias assessment following the guidance provided in the Cochrane Handbook for Systematic Reviews. ${ }^{22}$ This review will assess the possible evidence using the GRADE approach and will be reported in accordance with the PRISMA guidelines. The results of this systematic review will help dentists in clinical practice decide whether to provide additional PFA treatment for children who already use fluoride toothpaste daily. Limiting the toothpaste concentration to $1000 \mathrm{ppm}$ or more may lead to a small number of studies being included, but a more precise answer can be obtained.

Several studies in the past 5 years have shown little justification for the use of PFA combined with toothpaste, resulting in a negligible improvement in efficacy. ${ }^{31-34}$ The combined use of regular fluoride toothpaste with PFA may have no greater effect than regular fluoride toothpaste used alone. We plan to perform subgroup analyses of different interventions, different levels of caries risk and different follow-up times. First, different interventions will affect the efficacy of caries prevention. ${ }^{21}$ Second, previous literature has shown a correlation between different levels of caries risk and anticaries effects, which makes the level of caries risk another potential factor. ${ }^{35}$ In addition, different follow-up times can lead to different research results. ${ }^{36}$

The data regarding the necessity of additional PFA will provide guidance to professionals about the efficacy and adverse effects of PFA and consequently facilitate clinical decisions. This study may also identify areas of interest for future investigations.

\section{Author affiliations}

${ }^{1}$ Department of Paediatric Dentistry, Hubei-MOST KLOS \& KLOBM, School \& Hospital of Stomatology, Wuhan University, Wuhan, China

'Library, Hubei-MOST KLOS \& KLOBM, School \& Hospital of Stomatology, Wuhan University, Wuhan, China

${ }^{3}$ Wuhan Children's Hospital (Wuhan Maternal and Child Healthcare Hospital), Tongji Medical College, Huazhong University of Science and Technology, Wuhan, China ${ }^{4}$ Centre for Evidence-Based Stomatology, Hubei-MOST KLOS \& KLOBM, School \& Hospital of Stomatology, Wuhan University, Wuhan, China

${ }^{5}$ Division of Dentistry, School of Medical Sciences, Faculty of Biology, Medicine and Health, The University of Manchester, Manchester Academic Health Science Centre, Manchester, UK

Contributors $L Y$ and $X Y$ conceived of this review. $L Y$ and $X Y$ developed the search strategy and drafted the manuscript. YL, JL, FH and GS critically revised the manuscript. GS is the guarantor. All authors participated in the determination of eligibility criteria, the risk of bias assessment strategy and data extraction methods. All authors read and approved the final manuscript. 
Funding This work was supported by Hubei Provincial Natural Science Foundation of China, Grant/Award Number: 2015CFB258; Wuhan Young and Middle-aged Medical Talents Training Programme (№. (2019)87).

Disclaimer The funding sources had no involvement in the study design, collection, analysis and interpretation of data, preparation of the manuscript, or in the decision to publish.

Competing interests None declared.

Patient and public involvement Patients and/or the public were not involved in the design, or conduct, or reporting, or dissemination plans of this research.

Patient consent for publication Not required.

Provenance and peer review Not commissioned; externally peer reviewed.

Open access This is an open access article distributed in accordance with the Creative Commons Attribution Non Commercial (CC BY-NC 4.0) license, which permits others to distribute, remix, adapt, build upon this work non-commercially, and license their derivative works on different terms, provided the original work is properly cited, appropriate credit is given, any changes made indicated, and the use is non-commercial. See: http://creativecommons.org/licenses/by-nc/4.0/.

\section{ORCID iDs}

Fang Hua http://orcid.org/0000-0002-2438-5924

Guangtai Song http://orcid.org/0000-0002-5416-1665

\section{REFERENCES}

1 Selwitz RH, Ismail AI, Pitts NB. Dental caries. Lancet 2007;369:51-9.

2 Walsh T, Worthington HV, Glenny A-M, et al. Fluoride toothpastes of different concentrations for preventing dental caries. Cochrane Database Syst Rev 2019;2.

3 dos Santos APP, Nadanovsky P, de Oliveira BH. A systematic review and meta-analysis of the effects of fluoride toothpastes on the prevention of dental caries in the primary dentition of preschoo children. Community Dent Oral Epidemiol 2013;41:1-12

4 Toumba KJ, Twetman S, Splieth C, et al. Guidelines on the use of fluoride for caries prevention in children: an updated EAPD policy document. Eur Arch Paediatr Dent 2019;20:507-16.

5 Guideline on fluoride therapy. Pediatr Dent 2016;38:181-4.

6 KJA-Ohoo T, Twetman S, Splieth C, et al. Guidelines on the use of fluoride for caries prevention in children: an updated EAPD policy document. European Archives of Paediatric Dentistry. 1996-9805 (Electronic)).

7 Agouropoulos A, Twetman S, Pandis N, et al. Caries-preventive effectiveness of fluoride varnish as adjunct to oral health promotion and supervised tooth brushing in preschool children: a double-blind randomized controlled trial. J Dent 2014;42:1277-83.

8 Bal IS, Dennison PJ, Evans RW. Dental fluorosis in the blue Mountains and Hawkesbury, New South Wales, Australia: policy implications. J Investig Clin Dent 2015;6:45-52.

9 Wong MC, Glenny A-M, Tsang BW, et al. Topical fluoride as a cause of dental fluorosis in children. Cochrane Database Syst Rev 2010:CD007693.

10 Schwendicke F, Splieth $\mathrm{CH}$, Thomson WM, et al. Cost-effectiveness of caries-preventive fluoride varnish applications in clinic settings among patients of low, moderate and high risk. Community Dent Oral Epidemiol 2018;46:8-16.

11 American Dental Association Council on Scientific Affairs. Professionally applied topical fluoride: evidence-based clinical recommendations. J Am Dent Assoc 2006;137:1151-9.

12 Pollick $\mathrm{H}$. The role of fluoride in the prevention of tooth decay. Pediatr Clin North Am 2018:65:923-40.

13 Azarpazhooh A, Main PA. Fluoride varnish in the prevention of dental caries in children and adolescents: a systematic review. Hawaii Dent J 2009;40:6-17.
14 Detsomboonrat P, Trairatvorakul C, Pisarnturakit PP. Similar 1-year caries increment after use of fluoride or non-fluoride toothpaste in infants and toddlers. Fluoride 2016;49:313-26.

15 Ripa LW. A critique of topical fluoride methods (dentifrices, mouthrinses, operator-, and self-applied gels) in an era of decreased caries and increased fluorosis prevalence. J Public Health Dent 1991:51:23-41.

16 Marinho VCC, Higgins JPT, Sheiham A, et al. Combinations of topical fluoride (toothpastes, mouthrinses, gels, varnishes) versus single topical fluoride for preventing dental caries in children and adolescents. Cochrane Database Syst Rev 2004:CD002781.

17 Marinho VCC, Worthington HV, Walsh T, et al. Fluoride varnishes for preventing dental caries in children and adolescents. Cochrane Database Syst Rev 2013:CD002279.

18 Marinho VCC, Worthington HV, Walsh T, et al. Fluoride gels for preventing dental caries in children and adolescents. Cochrane Database Syst Rev 2015;13.

19 Marinho VCC, Higgins JPT, Sheiham A, et al. One topical fluoride (toothpastes, or mouthrinses, or gels, or varnishes) versus another for preventing dental caries in children and adolescents. Cochrane Database Syst Rev 2004:CD002780.

20 Marinho VCC, Higgins JPT, Logan S, et al. Topical fluoride (toothpastes, mouthrinses, gels or varnishes) for preventing dental caries in children and adolescents. Cochrane Database Syst Rev 2003:CD002782.

21 Cooper AM, O'Malley LA, Elison SN, et al. Primary school-based behavioural interventions for preventing caries. Cochrane Database Syst Rev 2013:CD009378.

22 Higgins JP, Green S. Cochrane Handbook for systematic reviews of interventions version 5.1.0, 2011. http://handbook-5-1.cochrane.org/

23 Marinho VCC, Worthington HV, Walsh T, et al. Fluoride varnishes for preventing dental caries in children and adolescents. Cochrane Database Syst Rev 2013;7:CD002279.

24 Marinho VCC, Chong LY, Worthington HV, et al. Fluoride mouthrinses for preventing dental caries in children and adolescents. Cochrane Database Syst Rev 2016;7:CD002284.

25 Higgins JPT, Thompson SG, Deeks JJ, et al. Measuring inconsistency in meta-analyses. BMJ 2003;327:557-60.

26 Huedo-Medina TB, Sánchez-Meca J, Marín-Martínez F, et al. Assessing heterogeneity in meta-analysis: Q statistic or 12 index? Psychol Methods 2006;11:193-206.

27 American Academy of Pediatric Dentistry. Guideline on caries-risk assessment and management for infants, children, and adolescents. Pediatr Dent 2013;35:E157-64.

28 Egger M, Davey Smith G, Schneider M, et al. Bias in meta-analysis detected by a simple, graphical test. BMJ 1997;315:629-34.

29 Hultcrantz M, Rind D, Akl EA, et al. The grade Working group clarifies the construct of certainty of evidence. J Clin Epidemiol 2017;87:4-13.

30 Guyatt GH, Oxman AD, Schünemann HJ, et al. Grade guidelines: a new series of articles in the Journal of clinical epidemiology. J Clin Epidemiol 2011;64:380-2.

31 Braun PA, Quissell DO, Henderson WG, et al. A cluster-randomized, community-based, Tribally delivered oral health promotion trial in Navajo head start children. J Dent Res 2016;95:1237-44.

32 Anderson M, Dahllöf G, Soares FC, et al. Impact of biannual treatment with fluoride varnish on tooth-surface-level caries progression in children aged 1-3 years. J Dent 2017;65:83-8.

33 Anderson M, Dahllöf G, Twetman S, et al. Effectiveness of early preventive intervention with Semiannual fluoride Varnish application in toddlers living in high-risk areas: a stratified cluster-randomized controlled trial. Caries Res 2016;50:17-23.

34 Tickle M, O'Neill C, Donaldson M, et al. A randomized controlled trial of caries prevention in dental practice. J Dent Res 2017;96:741-6.

35 Azarpazhooh A, Main PA. Fluoride varnish in the prevention of dental caries in children and adolescents: a systematic review. J Can Dent Assoc 2008;74:73-9.

36 van Rijkom HM, Truin GJ, van 't Hof MA. Caries-inhibiting effect of professional fluoride gel application in low-caries children initially aged 4.5-6.5 years. Caries Res 2004;38:115-23. 